\title{
Analysis of Single Cyclic Nucleotide-gated Channels in Olfactory Receptor Cells
}

\author{
Frank Zufall, ${ }^{1}$ Stuart Firestein, ${ }^{2}$ and Gordon M. Shepherd ${ }^{2}$ \\ 'Physiological Institute, Technical University Munich, 8000 Munich 40, Germany and ${ }^{2}$ Section of Neurobiology, Yale \\ University Medical School, New Haven, Connecticut 06510
}

In the accompanying article (Firestein et al., 1991b), we have demonstrated that odor- and cyclic nucleotide-sensitive channels exist at a low density in the dendritic membranes of isolated salamander olfactory receptor neurons. Here, we analyze the cyclic nucleotide sensitivity of these channels using the inside-out patch recording technique. Both CAMP and CGMP, at micromolar concentrations, are capable of inducing channel openings. The biophysical parameters of channel activity are nearly the same in response to either ligand. The unitary conductance is about $45 \mathrm{ps}$, the reversal potential of single-channel currents is $+5 \mathrm{mV}$, and the $/ / V$ relation is linear over the range -80 to $+80 \mathrm{mV}$. The channel activity shows no obvious voltage dependence in divalent cation-free symmetrical solutions. The channel shows no desensitization, even to agonist exposures lasting $15 \mathrm{sec}$. Mean open time is about $1.5 \mathrm{msec}$; the closed time distribution is best fit by two exponentials with a fast time constant in the submillisecond range (ca. $0.15 \mathrm{msec}$ ) and a slower time constant in the millisecond range (ca. $1.5 \mathrm{msec}$ ). The only clear difference in the activity of the two ligands is in their affinity constants. The $\kappa_{1 / 2}$ for CAMP is $20 \mu \mathrm{M}$; that for cGMP is $4 \mu \mathrm{M}$. In both cases, the Hill coefficient is greater than 2 , suggesting that channel opening requires the cooperative action of three ligand molecules.

In the accompanying article (Firestein et al., 1991b), we have demonstrated that an ionic channel found in patches of membrane from the dendrite and soma of isolated salamander olfactory receptor neurons is responsible for generating the odorsensitive current and that this same channel is activated by cyclic nucleotides intracellularly. In this report, we make further use of this preparation to investigate the behavior of single channels in excised patches of membrane from these same regions. This has enabled us to characterize the channel at a new level of analysis.

In addition to the unique interest for understanding olfactory transduction, investigation of this channel is motivated by other concerns as well. The channel is one of only two known to be directly gated by cyclic nucleotides (Hockberger and Swandulla,

\footnotetext{
Received Apr. 3, 1991; revised June 12, 1991; accepted June 20, 1991.

This work was supported by U.S. Public Health Service Grants DC-00086-24 and NS 101074-18 (G.M.S.) from the National Institutes of Health, Grant N0001490-J-1432 (S.F.) from the Office of Naval Research, and Deutsche Forschungsgemeinschaft (F.Z.)

Correspondence should be addressed to Stuart Firestein, Section of Neurobiology, Yale University Medical School, 333 Cedar Street, New Haven, CT 06510.

Copyright (C) 1991 Society for Neuroscience $0270-6474 / 91 / 113573-08 \$ 05.00 / 0$
}

1987), the other being the light-sensitive and cGMP-gated channel of vertebrate photoreceptors (Fesenko et al., 1985; Matthews, 1987). Comparison of the amino acid sequence of various versions of the cloned olfactory and visual channels reveals 60 $90 \%$ homology between them (Kaupp et al., 1989; Dhallan et al., 1990; Ludwig et al., 1990; Goulding et al., 1991), leading to suggestions that these two channels, and perhaps others yet to be discovered, constitute a new family of ligand-gated, ion channel receptors (Jan and Jan, 1990; Maelicke, 1990). Their electrophysiological behavior also appears quite similar, although it is notable that single photoreceptor channels have been recorded (Haynes et al., 1986), but in the olfactory receptor, recordings had been limited to the summed macroscopic current due to many channels (Nakamura and Gold, 1987) until the accompanying study (Firestein et al., 1991b).

One important difference between the olfactory and visual channels is their sensitivity to cyclic nucleotides. The photoreceptor channel is virtually insensitive to all cyclic nucleotides besides $3^{\prime}, 5^{\prime}$-cGMP (Fesenko et al., 1985), whereas the olfactory channel appears to respond to both cAMP and cGMP (Nakamura and Gold, 1987; Dhallan et al., 1990; Firestein et al., 1991b). The molecular mechanism responsible for this difference in selectivity remains unknown.

There is also some controversy regarding differences in affinity for the two cyclic nucleotides. In some preparations, there appears to be little difference (Goulding et al., 1991; Nakamura and Gold, 1987), but in others there may be as much as a 3050-fold greater sensitivity to cGMP (Dhallan et al., 1990). It is not clear whether these discrepancies are due to real variations between channels from different species, or to differences in the preparations or between cloned and native channels.

Resolution of these issues may require a more sensitive measure of activity than the summed behavior of a large population of channels. Analysis of the effects of cyclic nucleotides on the behavior of single channels could reveal important differences between the olfactory and visual channels. It would also be useful to have single-channel records from native channels available for comparison with similar observations from cloned channels. Finally, biophysical analysis is important since the channel plays a critical role in olfactory transduction. Its activity represents both the final step of the molecular transduction enzymc cascadc and the first step in the generation of the sensory electrical signal.

\section{Materials and Methods}

Preparation of the cells was the same as in the accompanying article (Firestein et al., 1991b). The normal Ringer's solution was the same, but once a seal was formed the bath was changed to a $\mathrm{Ca}^{2+}$-free Ringer's 
A
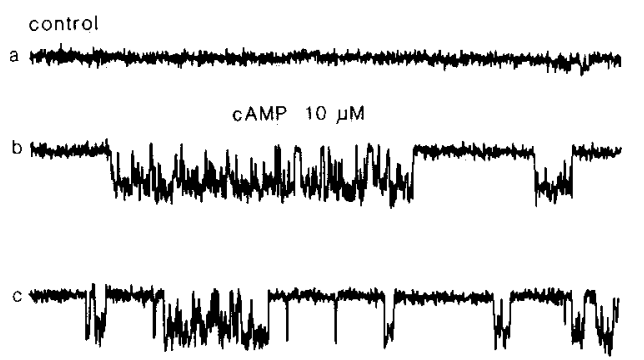

Figure 1. Inside-out patches from dendritic membrane of isolated olfactory receptor neuron. Control recording before $(a)$ and after $(e)$ application of agonists. Examples of single-channel events evoked by the continuous application of $10 \mu \mathrm{M}$ CAMP $(A, b-d)$ and $2 \mu \mathrm{M}$ cGMP $(B, b-d)$. IIolding potential, $-60 \mathrm{mV}$. Bandwidth, 0-2.5 kHz. Sampling rate, $15 \mathrm{kHz}$.

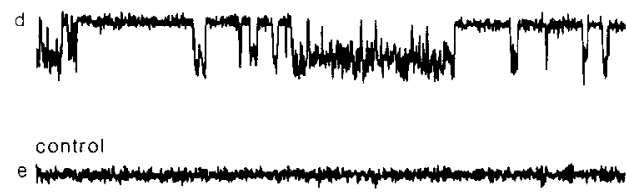

B

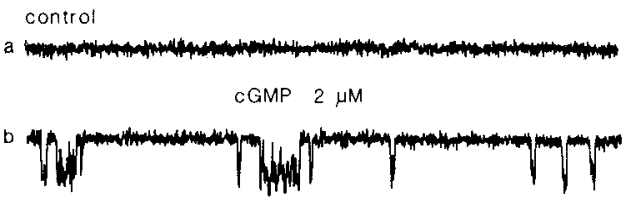

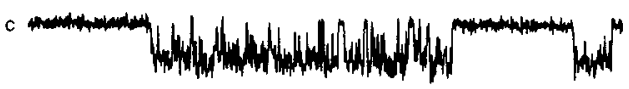

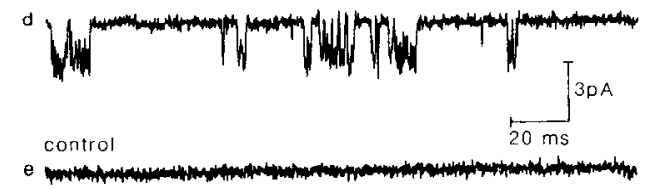

solution containing (in mM) $\mathrm{NaCl}, 117.5 ; \mathrm{CaCl}_{2}, 1$; EGTA, 10; HEPES, 10; pH 7.6. cAMP and cGMP (Sigma) were prepared daily as a stock solution in this $\mathrm{Ca}^{2+}$-free Ringer's solution at a concentration of $1 \mathrm{~mm}$ and diluted to working concentrations. The cyclic nucleotide solutions were delivered to the patch via a continuous flow system consisting of six separate capillary tubes. The system is similar to one described by Yellen (1982) and allows complete solution changes to be made in less than 1 sec.

Recordings were made using the inside-out configuration of the patch clamp (Hamill et al., 1981). Electrodes were pulled from thin-walled borosilicate glass (World Precision Instruments 150) fire polished to tips with resistances from 8 to $15 \mathrm{M} \Omega$ and coated with Sylgard. The pipette solution was the same as the $\mathrm{Ca}^{2+}$-free Ringer, so that both faces of the membrane patch were bathed in symmetrical solutions.

Single-channel currents were recorded to videotape using a List EPC-7 patch-clamp amplifier. For analysis, the signals were digitized and stored on an IBM 386 computer using pCLAMP software (Axon Instruments). Filter bandwidths and sampling rates are given below and in figure captions.

\section{Results}

\section{Recordings from inside-out patches}

Characteristic single-channel activity recorded from inside-out membrane patches is shown in Figure 1. The control records (top traces), in divalent-free symmetrical solutions with zero $\mathrm{K}^{+}$, showed no channel openings (Fig. $1 A, B$, traces a). When cAMP (10 $\mu \mathrm{M})$ was added to the bathing medium (i.e., the cytosolic membrane face of the patch), single-channel openings and bursts occurred. The $\mathrm{b}-\mathrm{d}$ traces in Figure $1 A$ show a sampling of this activity. The effects were fully and rapidly reversible upon returning to the cyclic nucleotide-free solution (Fig. $1 A, B$, traces e).

At negative holding potentials (inside with respect to outside), the currents were inward, and openings and closings were rapid. Recordings were stable for up to $45 \mathrm{~min}$, including several changes of bathing solutions. The patches generally were taken from the dendrite or knob area of the cell as described in the accompanying article (Firestein et al., 199 lb). In many cells, the dendrites were very short (less than $10 \mu \mathrm{m}$ ), and in some they were virtually nonexistent. Patches taken from all these regions contained similar channels. A total of 42 patches were analyzed, of which 29 showed single-channcl activity in response to cyclic nucleotides. Most possessed only a single channel; a few had up to three superpositions of channel openings.

The results with cGMP as the cyclic nucleotide were very similar, as shown in Figure $1 B$, traces a-e. Qualitatively, there were no significant differences from the type of channel activity seen with cAMP. A total of 11 patches were tested with both cyclic nucleotides, and all were sensitive to both. We concluded that cAMP and cGMP acted upon the same channel in our patches.

The temporal pattern of activity following exposure to cyclic nucleotides was further investigated by the use of a constant flow system of solution delivery as described in Materials and Methods. This system permitted the rapid and continuous application of stimulus solutions with known concentrations. As seen in Figure 2, application of cAMP at $100 \mu \mathrm{M}$ was followed immediately by the onset of channel activity. At this slow sweep speed, it was possible to discern at least two channels in this patch. The most significant aspect of this recording is the continued and sustained channel activity throughout the steady application of the cAMP, for over $13 \mathrm{sec}$ in this case. In five patches tested in this way, all showed the same pattern. A frequency plot of the same data is shown in Figure $2 C$, which also shows the continued sustained level of channel activity, and the multiple open states. Patches exposed to cGMP $(n=4)$ gave similar results.

Similar experiments were performed in the presence of $1 \mathrm{~mm}$ ATP $(n=3)$. In no case did we observe any effect of ATP on the open channel probability or the temporal pattern of cAMPinduced channel activity. Thus, these channels are activated directly by cyclic nucleotides and show no evidence for modulation by membrane-bound kinases.

These experiments suggest that the cyclic nucleotide-gated channel does not densensitize in the presence of continued exposure to cyclic nucleotide. This raises an interesting question concerning the relation between these microscopic currents and the macroscopic currents recorded in the whole-cell mode, which do show varying degrees of adaptation to plateau stimuli (Firestein et al., 1990; see Discussion). For experimental purposes, the lack of desensitization was a useful finding, because it permitted us to evaluate the properties of the channel under steadystate conditions of stimulation.

\section{Analysis of single channels}

Analysis of the single-channel activity under these conditions showed that the amplitude histogram was characteristically near- 


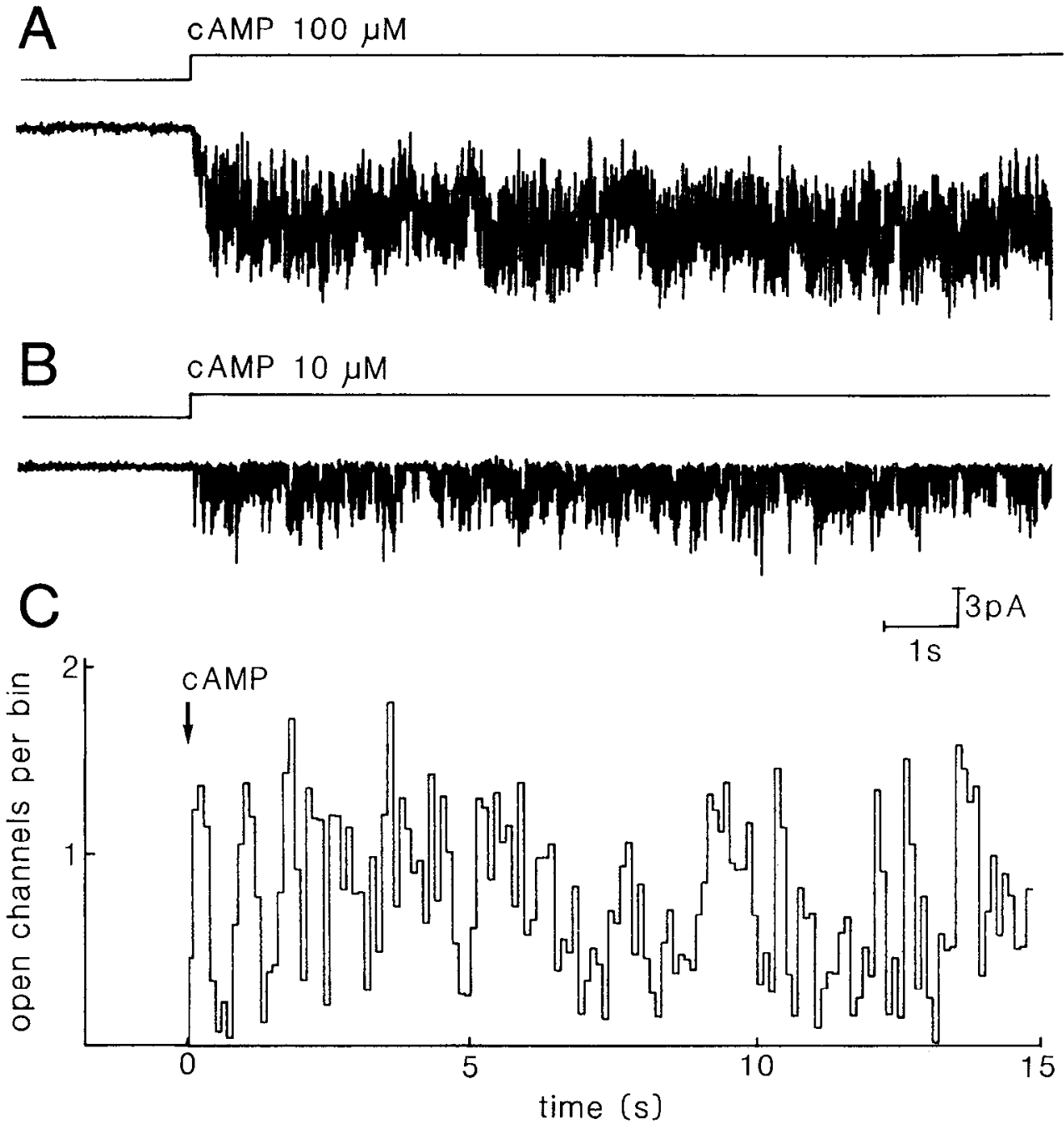

Figure 2. Single-channel activity on slow time scale after rapid application of high and low concentrations of cAMP to inside-out patches. Holding potential, $-60 \mathrm{mV}$. $A$, Fast application of $100 \mu \mathrm{M}$ cAMP evokes a nondesensitizing current composed of the simultaneous opening of at least five channels. $B$, In another patch, the opening of up to three channels can be seen during application of $10 \mu \mathrm{M}$ cAMP. Bandwidth, $0-500 \mathrm{~Hz}$. $C$, Evaluation of the number of open channels from the recording shown in $B$ over a time of 15 sec. As can be seen, there is no obvious desensitization. Bin width, $100 \mathrm{msec}$. monotonic. In the example of Figure $3 A$, at a holding potential of $-60 \mathrm{mV}$ a single peak is present at $2.9 \mathrm{pA}$ and is well fit by a single Gaussian curve. Analysis of five patches exposed to cAMP gave peak currents for single channels of -2.5 to -3.5 pA (mean, $-2.9 \pm 0.3 \mathrm{pA}$ ). Figure $3 D$ shows similar amplitude data for cGMP-treated channels. In this case, the peak channel amplitude is $-2.7 \mathrm{pA}$. For four patches tested with cGMP, the mean was $-2.8 \mathrm{pA}( \pm 0.35)$. In both instances, there is a small shoulder in the lower amplitude region of the curves, suggesting one or more subconductance states.

The open time of the channel was analyzed as in Figure 3, $B$ (cAMP) and $E$ (cGMP). Open time distributions for both cAMPand CGMP-stimulated channels were well fit by a single exponential. In Figure $3 B$, the mean open time was $1.9 \mathrm{msec}$. For six patches treated with cAMP, the mean was $1.7 \mathrm{msec}( \pm 0.25)$. Similar results were obtained from five channels treated with cGMP, although there was slightly more variability. Typical measurements are plotted in Figure $3 E$, where the mean open time is $1.3 \mathrm{msec}$. For the five patches, the mean was $1.5 \mathrm{msec}$ $( \pm 0.35)$.

Closed time distributions for both cAMP- and cGMP-treated channels are shown as Figure 3, $C$ and $F$, respectively. Both are shown on semi-log coordinates to facilitate the visualization of the two exponentials required for a reasonable fit of the data. The shorter time constant was $0.19 \mathrm{msec}$ and the longer was
$1.7 \mathrm{msec}$ for cAMP-driven channels. For cGMP, the two time constants were $0.22 \mathrm{msec}$ and $1.0 \mathrm{msec}$. The results comparing cAMP and cGMP are summarized in Table 1. It can be seen that there were no significant differences between the two cyclic nucleotides in single-channel conductance or opening and closing time constants revealed by these analyses.

The voltage sensitivity of the channel was analyzed by exposing patches with a single channel to saturating concentrations of cyclic nucleotide while setting the membrane patch at different holding potentials. Typical recordings at a few clamp potentials are shown in Figure $4 A$, and the current-voltage relation is shown in Figure $4 B$. Each point on the curve represents data from 3000 events at a given holding potential. As can be

\begin{tabular}{lll}
\hline $\begin{array}{l}\text { Table 1. Comparison of single-channel currents elicited by cAMP } \\
\text { and cGMP }\end{array}$ & cAMP & cGMP \\
\hline Single-channel conductance & $46 \mathrm{pS}$ & $44 \mathrm{pS}$ \\
Mean open time & $1.7 \mathrm{msec}$ & $1.5 \mathrm{msec}$ \\
Closed time & & \\
$\tau_{1}$ & $0.19 \mathrm{msec}$ & $1.7 \mathrm{msec}$ \\
$\tau_{2}$ & $0.22 \mathrm{msec}$ & $1.0 \mathrm{msec}$ \\
$K_{1 / 2}$ & $20 \mu \mathbf{M}$ & $4 \mu \mathrm{M}$ \\
\hline
\end{tabular}


A
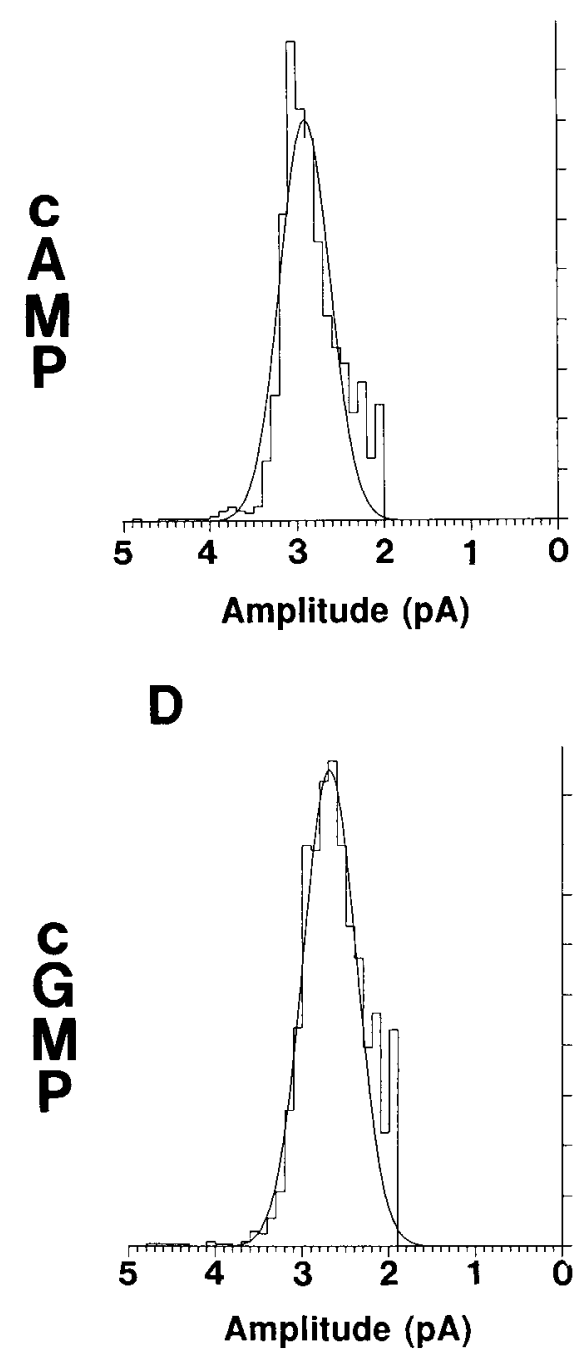
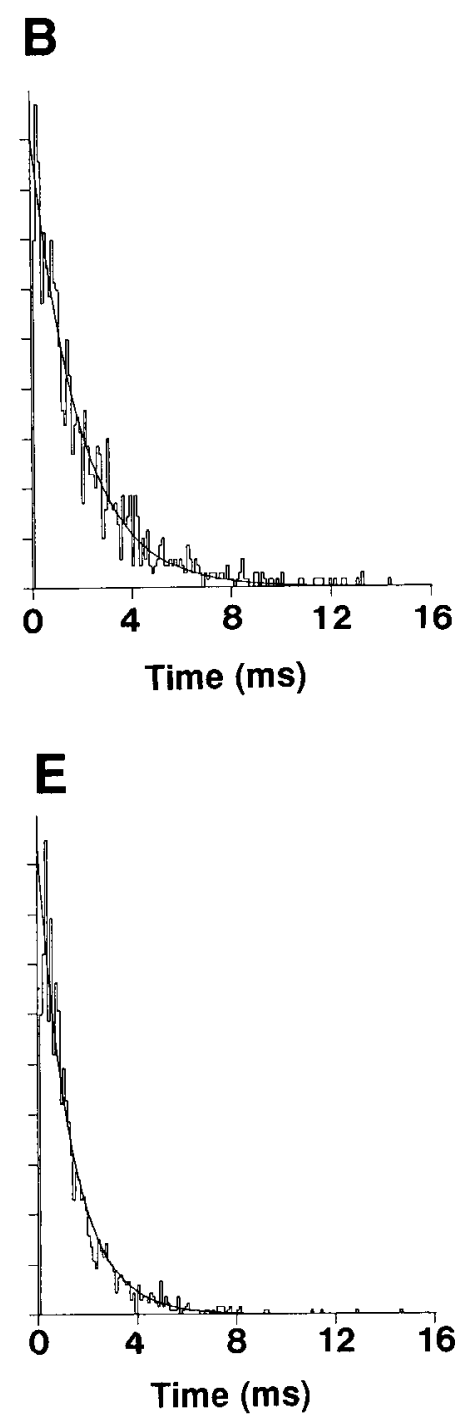
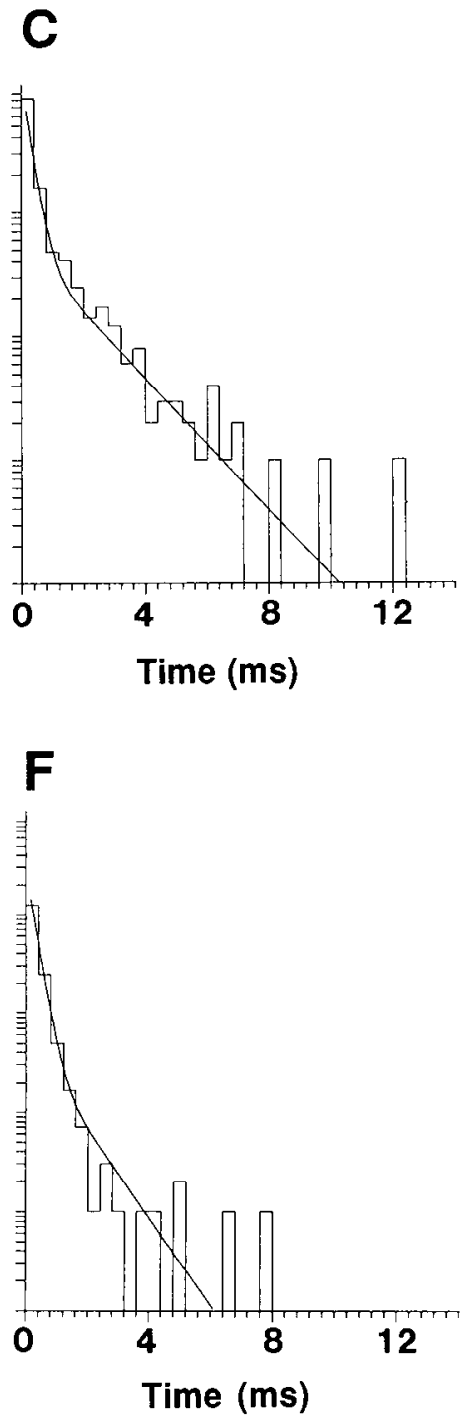

Figure 3. Analysis of channel kinetics for the two ligands. A, Amplitude histogram for the cAMP-elicited channel activity. Each division on the ordinate scale is 20 counts, total of 2000 events. Peak, $-2.9 \mathrm{pA}$; holding potential, $-60 \mathrm{mV}$. $B$, Open time distribution for the cAMP-evoked channel activity. Single exponential fit has a time constant of $1.89 \mathrm{msec}$. Bin width, $0.2 \mathrm{msec}$. Data includes 2000 events. $C$, Closed time distribution is best fit by two exponentials with time constants $0.19 \mathrm{msec}$ and $1.7 \mathrm{msec}$. The distribution is plotted on semi-log coordinates to facilitate visualization of the double exponential fit. Bin width, $0.4 \mathrm{msec}$. $D$, Amplitude histogram for the cGMP-elicited activity is well fit by a single Gaussian with a peak at $-2.7 \mathrm{pA}$. Holding potential, $-60 \mathrm{mV}$. Total number of events was 1600 ; each division on the ordinate is 20 counts. $E$, Open time distribution for cGMP-induced activity is well fit by a single exponential with time constant 0.9 msec. Based on 1600 events; bin width, $0.2 \mathrm{msec}$. $F$. Closed time distribution for the cGMP-induced activity is best fit with two exponentials of time constants $0.22 \mathrm{msec}$ and $1.0 \mathrm{msec}$. The data, 1600 counts, are plotted on semi-log coordinates to facilitate visualization of the two exponentials.

seen, the $I / V$ plot was nearly linear, and the reversal potential was $+5 \mathrm{mV}$, indicating that the channel conductance is largely due to passive ion flow. The only suggestion of a voltage sensitivity was seen at relatively hyperpolarized (inside vs. outside) membrane potentials, where there was slight inward rectification. The data shown in Figure 4 are for cAMP, but similar results were seen for both cyclic nucleotides (cAMP, $n=6$; cGMP, $n=4$ ).

Another test for voltage dependence was the open probability of the channel at different holding potentials. Over the range $-100 \mathrm{mV}$ to $+100 \mathrm{mV}$ membrane potential, the mean open probability was relatively constant; in the example of Figure $4 B$ (inset), the mean open probability was between 0.61 and 0.72 over the $200 \mathrm{mV}$ test range during exposure to $50 \mu \mathrm{M}$ cAMP. Similar to the $I / V$ plots above, these results gave no evidence of a significant voltage dependence under these recording conditions ( $\mathrm{K}$ conductances blocked; $0 \mathrm{Mg}^{2+}, 10^{-7} \mathrm{Ca}^{2+}$ ).

\section{Concentration dependence of channel activation}

The concentration dependence of the channcl activity was analyzed by examining the dose-response relation over a concentration range from 0 to $1 \mathrm{~mm}$ cyclic nucleotide. Typical recordings over this range for cAMP and cGMP are shown in Figure 5. In control conditions with zero cyclic nucleotide concentration and the holding potential at $-50 \mathrm{mV}$, there were no openings. At saturating concentrations, up to four superpositions of single inward currents could be observed. The dose-response curves for these data are plotted in Figure 6, where the points are fit by the Hill equation. Note the generally parallel plots for the two nucleotides, and the shift of the cGMP curve to the left, 
A

$80 \mathrm{mV}$

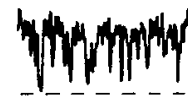

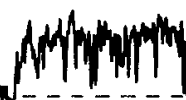

$5 \mathrm{mV}$

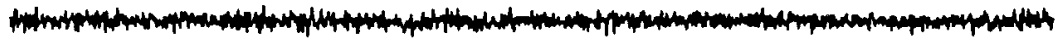

$-40 \mathrm{mV}$

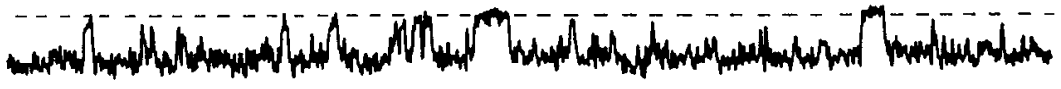

$-60 \mathrm{mV}$
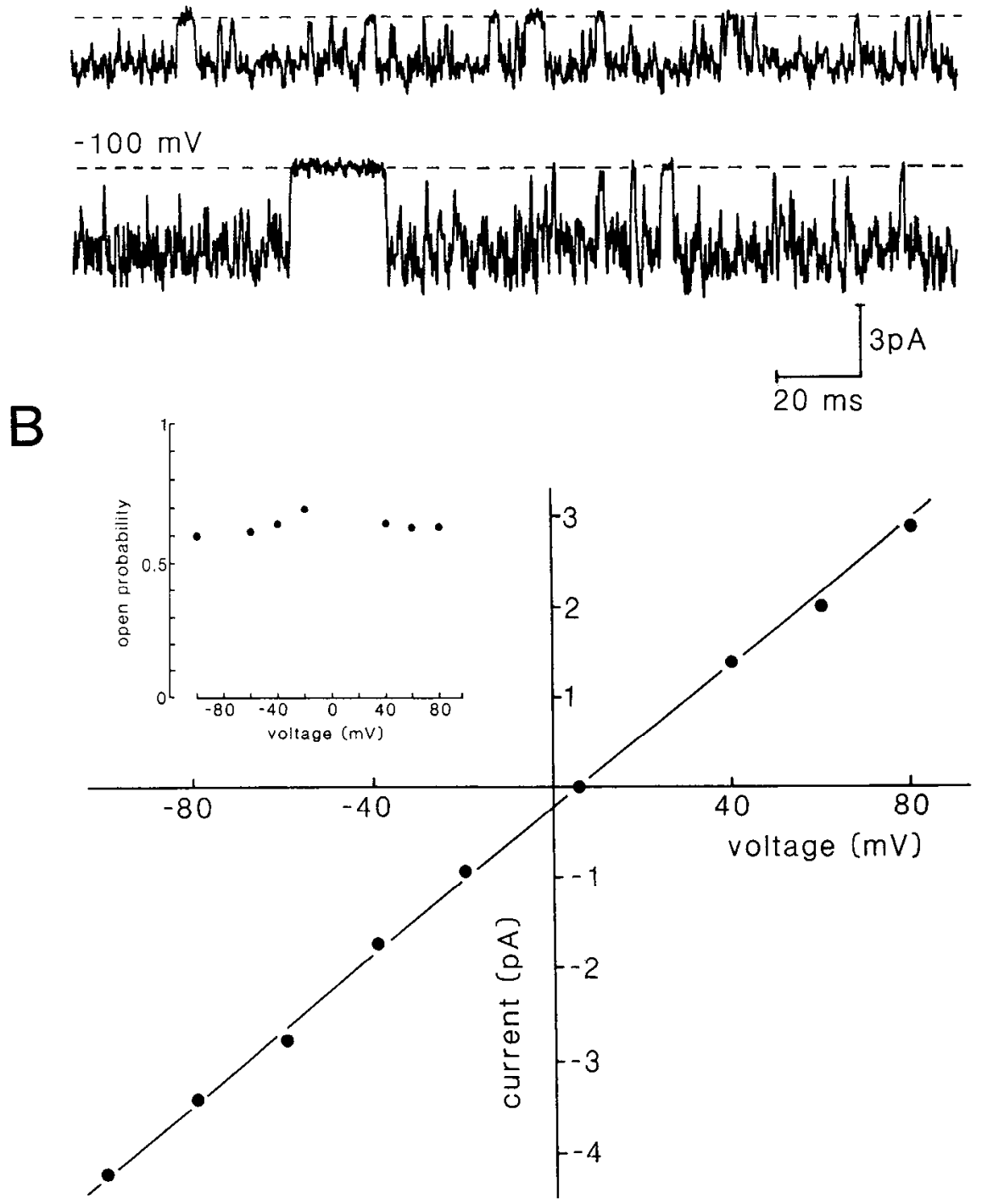

Figure 4. Voltage dependence of the cAMP-activated channel during continuous application of the saturating concentration of $0.1 \mathrm{~mm}$ cAMP. Only one channel is present in this patch. $A$, Examples of single-channel openings at different holding potentials as indicated for each trace. The closed state is marked by the broken line. $B, I / V$ relation of the main open state. The points were connected by a straight line, the slope of which gives a single channel conductance of $45 \mathrm{pS}$. The reversal potential is $+5 \mathrm{mV}$. Each point represents 3000 events from the recording shown in 1. The inset shows the dependence of the open probability on the membrane potential. No obvious voltage dependence is present.

indicating a slightly higher sensitivity of the channel to cGMP. The Hill coefficients were 2.1 for both cGMP and cAMP. The $K_{1 / 2}$ was $4 \mu \mathrm{M}$ for cGMP and $20 \mu \mathrm{M}$ for cAMP. Similar results were obtained in three other experiments.

\section{Discussion}

Excising patches of membrane from the single thick dendrite of isolated olfactory receptor neurons has allowed us to obtain recordings of single-channel activity elicited by the application of cyclic nucleotides. The existence of these channels had been inferred from whole-cell recordings of macroscopic currents (Kurahashi, 1989; Firestein et al., 1990) and from excised patches of ciliary membrane (Nakamura and Gold, 1987); however, analysis at the level of the single channel has not previously been possible due to the high numbers of simultaneously activated channels in these preparations. That the channels derived 
A

control

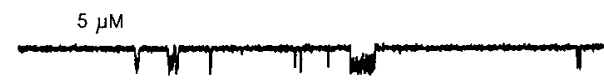

$10 \mu \mathrm{M}$

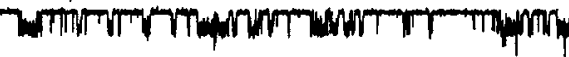

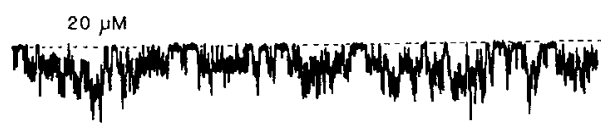

Figure 5. Concentration dependence of an inside-out patch at $-60 \mathrm{mV}$ to the continuous application of $\operatorname{cAMP}(A)$ and $c G M P(B)$. The numbers above each trace refer to the concentration of ligand. The broken line marks the closed state.

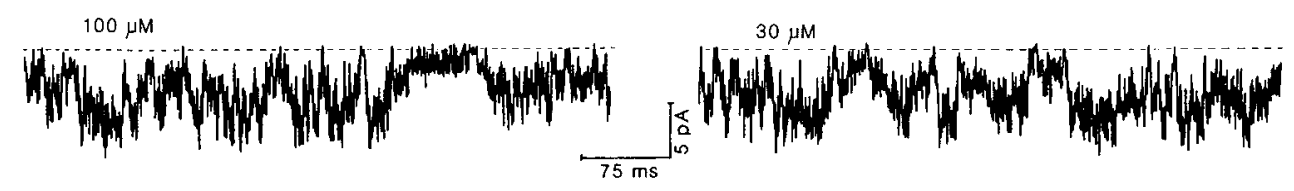

from dendritic membrane are the same as those previously implicated in the odor-sensitive conductance pathway was demonstrated in the accompanying article (Firestein et al., 1991b). Here, we have begun the task of characterizing their molecular behavior.

The finding that the channels are sensitive to both cAMP and cGMP is consistent with previous observations of whole-cell currents (Trotier and MacLeod, 1986; Suzuki, 1989; Kurahashi, 1990; Firestein et al., 1991a), ciliary currents (Nakamura and Gold, 1987), and currents recorded from functionally expressed cloned channels (Dhallan et al., 1990; Iudwig et al., 1990) in which both nucleotides were effective gating ligands. In this previous work, there was general agreement that the channel is more sensitive to CGMP than CAMP, but the precise ratio of the affinities varied among the several preparations. In native channels from bullfrog, Nakamura and Gold (1987) measured a cGMP:cAMP sensitivity ratio of 1.7 , whereas Dhallan et al. (1990), in cloned mammalian channels, reported a ratio of 30 .

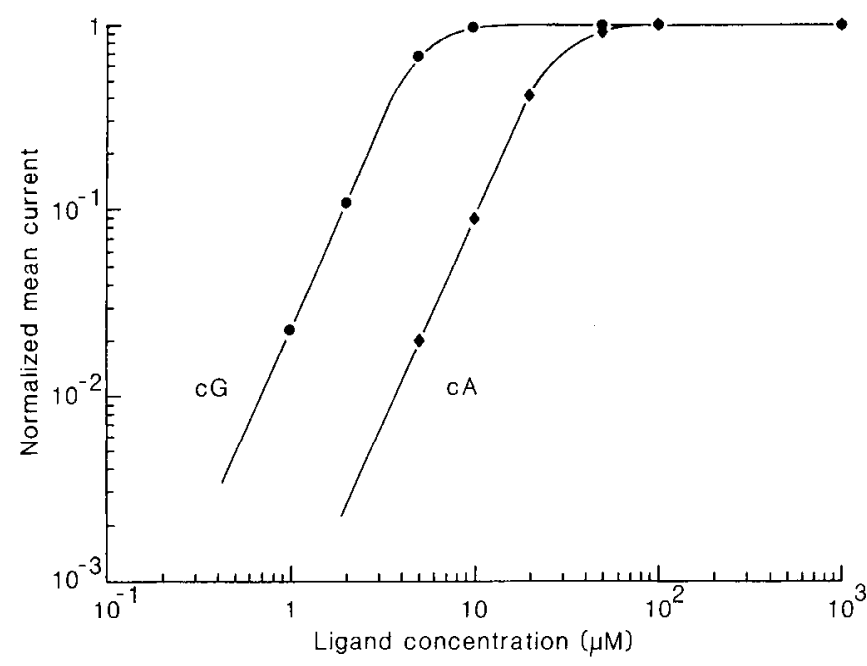

Figure 6. Dose-response curves for the data in Figure 5. The normalized mean current was plotted against ligand concentration in a double logarithmic plot. The points were fit by the Hill equation. The values of $K_{1 / 2}$ were $20 \mu \mathrm{M}$ for cAMP and $4 \mu \mathrm{M}$ for cGMP; $n$ was 2.1 for both.
Recently, Goulding et al. (1991), in a channel derived from catfish olfactory neurons, determined that the $K_{1 / 2}$ for both nucleotides was the same, but at $95 \mu \mathrm{M}$ was somewhat higher than in other preparations. We find that the $K_{1 / 3}$ for cGMP is five times lower than that for cAMP ( 4 vs. $20 \mu \mathrm{M}$ ).

More critically, the source of the variation is primarily in the sensitivity to cAMP. The $K_{1 / 2}$ values reported were $2.4 \mu \mathrm{M}$ (native channel, bullfrog), $39 \mu \mathrm{M}$ (cloned channel, rat), and $20 \mu \mathrm{M}$ in our single channels from salamander, a range of more than one order of magnitude. [Kurahashi (1990) reported a much larger $K_{1 / 2}$ of $300 \mu \mathrm{M}$, but this was obtained using whole-cell recordings of different cells with different concentrations of cAMP in the recording pipettes.] By contrast, the values for cGMP in the different preparations were, respectively, $1.6,1.2$, and $4 \mu \mathrm{M}$, only a twofold range of variation. Since there is a very active odor-sensitive adenylate cyclase present in olfactory neurons (Pfeuffer et al., 1989; Bakalyar and Reed, 1990) and no detectable odor-sensitive guanylate cyclase activity (Shirley et al., 1986), it is generally assumed that CAMP is the main second messenger in these cells. The role of cGMP, if there is any, is unclear. This makes it all the more surprising that the $K_{1 / 2}$ values for cAMP vary over more than an order of magnitude while the cGMP values are within a twofold range.

The different values of the $K_{1 / 2}$ were the only substantial difference that we were able to detect between the action of the two nucleotides. The mean channel conductance, the mean open time, and the opening and closing time constants were identical within experimental variability. Thus, once a cyclic nucleotide is bound, the behavior of the channel is the same.

Regarding the level of cooperativity in ligand binding, our results are in general agreement with previous findings. We found a Hill coefficient, for both CGMP and CAMP, of 2.1, compared to 1.8 in both the native (Nakamura and Gold, 1987) and cloned channel (Dhallan et al., 1990). The somewhat higher Hill coefficient obtained from single-channel measurements is also seen in recordings from the photoreceptor channel (Haynes et al., 1986). Apparently, measurements derived from macroscopic currents tend to underestimate the level of cooperativity, but the reasons for this are not clear (Yau and Baylor, 1989).

The electrical properties of the single channel are also similar to those measured for summed currents. The $I / V$ relation is linear and the reversal potential is near $0 \mathrm{mV}$. Additionally, we 
find that the activity of single channels recorded in divalent cation-free symmetrical solutions is not voltage dependent. The effects of divalent ions on this channel are similar to those seen in native channcls and in wholc-cell recordings. Those data were not shown here but will be presented in a separate communication.

We note that the single-channel conductance determined here from excised patches is slightly larger than that measured in the previous experiments with on-cell patches. A similar difference was noted by Matthews and Watanabe (1987) in recordings from photoreceptors. Several reasons for the small discrepancies may be put forward. One is that the solutions in contact with the membrane in the two recording conditions are different. In the case of the on-cell patches, the extracellular and intracellular solutions are of different compositions, whereas the excised patches are bathed in symmetrical solutions containing only $\mathrm{Na}$ ions. Furthermore, the solution facing the intracellular face of the membrane in the excised patch is strongly buffered for low $\mathrm{Ca}^{2+}$, probably more so than the normal cytosol in the on-cell patch.

An important finding in our results was that long exposures of single channels to even saturating concentrations of cyclic nucleotide did not result in any detectable desensitization. By contrast, recordings from intact cells show strong desensitization to both odors and elevated intracellular cAMP (Firestein et al., 1990, 1991a; Kurahashi, 1990). This suggests that the mechanism of desensitization requires some additional intracellular factors, perhaps a kinase, which is lost when the membrane patch is excised. In any case, channel activity alone is not sufficient to cause any modulation of the mean open time.

From the data presented here, it is possible to estimate both the density of channels existing on the cilia and also the percentage of channels activated by a saturating pulse of odor. In divalent ion-free solutions, we found a single-channel current amplitude of around $1 \mathrm{pA}$ with a driving force of $25 \mathrm{mV}$. Nakamura and Gold (1987), under similar conditions, were able to elicit a $240 \mathrm{pA}$ current from a small patch of ciliary membrane Their patch pipettes were smaller than normal because of the diameter of the cilia (about $0.25 \mu \mathrm{m}$ ), and so the estimated membrane area in the patch would be about $0.2 \mu \mathrm{m}^{2}$. This gives a value of 1200 channels $/ \mu \mathrm{m}^{2}$ in the ciliary membrane. It is difficult to measure the exact area of the membrane drawn into a patch pipette, but since the electrodes used in this case possessed exceptionally small apertures leading to less likelihood of bleb formation (Milton and Caldwell, 1990), we believe that these values are reasonable. By comparison, similar estimates of cGMP channels in rod outer segment are around 500-800 channels $/ \mu \mathrm{m}^{2}$ (Zimmerman et al., 1985; Haynes et al., 1986; Matthews and Watanabe, 1987), or between $1 / 2$ and $2 / 3$ that of the olfactory receptor. Nakamura and Gold (1988) reported an estimated single-channel density that ranged between 450 and 7000 channels $/ \mu \mathrm{m}^{2}$, but their measured single-channel conductance was $25-30 \mathrm{pS}$ as compared to the $46 \mathrm{pS}$ measured here. In freeze-fracture electron micrographs of the olfactory cilia, intramembranous particles exist at a density of nearly 2500 / $\mu \mathrm{m}^{2}$ (Menco, 1980), although their exact identity is unknown.

The response amplitude of the intact olfactory cell to a strong pulse of odor is variable but may be as large as $600 \mathrm{pA}$ at a membrane holding potential of $-60 \mathrm{mV}$ (Firestein and Werblin, 1989; Kurahashi, 1989; Firestein et al., 1990). At this potential, an individual channel is capable of conducting $2.5 \mathrm{pA}$ of current, suggesting the minimum activation of perhaps 250 channels. If the cilia are $0.25 \mu \mathrm{m}$ in diameter (Rafols and Getchell, 1983) and approximately $40 \mu \mathrm{m}$ in length, then their approximate surface area is $31 \mu \mathrm{m}^{2}$. A typical cell may possess up to 10 cilia, for a total number of channel molecules approaching half a million. Even if the conductance of the single channel is 2 orders of magnitude smaller in the presence of physiological levels of $\mathrm{Ca}^{2+}$ (as is the case for the photoreceptor channel), this means that only about $5-6 \%$ of the available channels are activated by even a saturating pulse of odor. While these calculations should be considered as rough estimates because of the variability in membrane patch size, they seem reasonable for a sensory receptor mechanism; for example, in rod photoreceptors only 1$2 \%$ of the available cGMP-activated channels are open in the dark (Yau and Baylor, 1989).

\section{References}

Bakalyar HA, Reed RR (1990) Identification of a specialized adenylyl cyclase that may mediate odorant detection. Science 250:1403-1406.

Dhallan RS, Yau KW, Schrader KA, Reed RR (1990) Primary structure and functional expression of a cyclic nucleotide-activated channel from olfactory neurons. Nature 347:184-187.

Fesenko EE, Kolesnikov SS, Lyubarsky AL (1985) Induction by cyclic GMP of cationic conductance in plasma membrane of retinal rod outer segment. Nature 313:310-313.

Firestein S, Werblin F (1989) Odor-induced membrane currents in vertebrate olfactory receptor neurons. Science 244:79-82.

Firestein S, Shepherd GM, Werblin FS (1990) Time course of the membrane current underlying sensory transduction in salamander olfactory receptor neurones. J Physiol (Lond) 430:135-158.

Firestein S, Darrow B, Shepherd GM (1991a) Activation of the sensory current in salamander olfactory receptor neurons depends on a G-protein mediated cAMP second messenger system. Neuron 6:825-835.

Firestein S, Zufall F, Shepherd GM (1991b) Single odor-sensitive channels in olfactory receptor neurons are also gated by cyclic nucleotides. J Neurosci 11:3565-3572.

Goulding E, Ngai J, Chess A, Kramer R, Axel R, Seigelbaum S (1991) Cloning and characterization of an olfactory specific cyclic nucleotide gated cation channel. Biophys .I 59:391a.

Hamill OP, Marty A, Neher E, Sakmann B, Sigworth FJ (1981) Improved patch-clamp techniques for high-resolution current recording from cells and cell-free membrane patches. Pfluegers Arch 391:85100.

Haynes LW, Kay AR, Yau K-W (1986) Single cyclic GMP-activated channel activity in excised patches of rod outer segment membrane. Nature 321:66-70.

Hockberger PE, Swandulla D (1987) Direct ion channel gating: a new function for intracellular messengers. Cell Mol Neurobiol 7:229-236.

Jan LY, Jan YN (1990) A superfamily of ion channels. Nature 345: 672.

Kaupp UB, Tsutomu TN, Terada S, Bönigk W, Stühmer W, Cook NJ, Kangawa K, Matsuo H, Hirose T, Miyata T, Numa S (1989) Primary structure and functional expression from complementary DNA of the rod photoreceptor cyclic GMP-gated channel. Nature 342:762-766.

Kurahashi T (1989) Activation by odorants of cation-selective conductance in the olfactory receptor cell isolated from the newt. J Physiol (Lond) 419:177-192.

Kurahashi T (1990) The response induced by intracellular cyclic AMP in isolated olfactory receptor cells of the newt. J Physiol (Lond) 430: 355-371.

Ludwig J, Margalit T, Eisman E, Lancet D, Kaupp UB (1990) Primary structure of cAMP-gated channel from bovine olfactory epithelium. FEBS Lett 270:24-29.

Maelicke A (1990) The cGMP-gated channel of the rod photoreceptor-a new type of channel structure. Trends Biochem Sci 15:39-40.

Matthews G (1987) Single channel recordings demonstrate that cGMP opens the light sensitive ion channel of the rod photoreceptor. Proc Natl Acad Sci USA 84:299-302.

Matthews G, Watanabe S-I (1987) Properties of ion channels closed by light and opened by guanosine $3^{\prime}-5^{\prime}$-cyclic monophosphate in toad retinal rods. J Physiol (Lond) 389:691-716. 
Menco BPM (1980) Qualitative and quantitative freeze-fracture studies on olfactory nasal respiratory epithelial surfaces of frog, ox, rat and dog. II. Cell apices, cilia and microvilli. Cell Tissue Res 211:529.

Milton RL, Caldwell JH (1990) How do patch clamp seals form? A liquid bleb model. Pfluegers Arch 416:758-765.

Nakamura T, Gold GH (1987) A cyclic-nucleotide gated conductance in olfactory receptor cilia. Nature $325: 442-444$.

Nakamura T, Gold GH (1988) Single channel properties of the ciliary cyclic nucleotide -gated conductance. Chem Senses 13:723.

Pfeuffer E, Mollner S, Lancet D, Pfeuffer T (1989) Olfactory adenylyl cyclase. Identification and purification of a novel enzyme form. J Biol Chem 264:18803-18807.

Rafols JA, Getchell TV (1983) Morphological relations between the receptor neurons, sustenacular cells, and Schwann cells in the olfactory mucosa of the salamander. Anat Rec 206:87-101.
Shirley SG, Robinson CJ, Dickinson K, Aujla R, Dodd GH (1986) Olfactory adenylate cyclase of the rat. Biochem J 240:605-607.

Suzuki N (1989) Voltage- and cyclic nucleotide-gated currents in isolated olfactory receptor cells. In: Chemical senses, Vol 1 (Brand JG, Teeter JH, Cagan RH, Kare MR, eds), pp 469-494. New York: Dekker.

Trotier D, MacLeod P (1986) cAMP and cGMP open channels and depolarize olfactory receptor cells. Chem Senses 11:674.

Yau K-W, Baylor DA (1989) Cyclic GMP-activated conductance of retinal photoreceptor cells. Annu Rev Ncurosci 12:289-328.

Yellen $\mathrm{G}$ (1982) Single $\mathrm{Ca}^{++}$activated nonselective cation channels in neuroblastoma. Nature 296:357-359.

Zimmerman AL, Yamanaka G, Eckstein F, Baylor DA (1985) Interaction of hydrolysis-resistant analogs of cyclic GMP with the phosphodiesterase and light-sensitive current of retinal rod outer segments. Proc Natl Acad Sci USA 82:8813-8817. 